\title{
Numerical Solutions of the Forced Korteweg-de Vries-Burgers Equation
}

\author{
${ }^{1}$ K.G. Tay, ${ }^{2}$ W.K. Tiong, ${ }^{3}$ Y.Y. Choy and ${ }^{4}$ C.T. Ong \\ ${ }^{1}$ Department of Communication Engineering, Faculty of Electrical and Electronic Engineering, \\ Universiti Tun Hussein Malaysia (UTHM), Parit Raja, Malaysia E-mail:tay@uthm.edu.my \\ ${ }^{2}$ Computational Science and Mathematics, University of Malaysia Sarawak, Sarawak, Malaysia \\ ${ }^{3}$ Department of Mathematics, Faculty of Applied Science and Technology, \\ Universiti Tun Hussein Malaysia (UTHM), Parit Raja, Malaysia \\ ${ }^{4}$ Department of Mathematical Science, Faculty of Science, Universiti Teknologi Malaysia (UTM) \\ Parit Raja, Malaysia
}

\begin{abstract}
In this study, we solved the forced Korteweg-de Vries-Burgers (FKdVB) with variable coefficient arises in nonlinear wave propagation in an elastic tube with a symmetrical stenosis filled with varible viscocity fluid by two numerical methods, namely method of lines and finite-difference method. We then compared both numerical solutions with its progressive wave solution. Both methods solve the FKdVB equation with maximum absolute errors of $10^{-7}$.
\end{abstract}

Key words: Forced Korteweg-de vries-Burgers, Korteweg-de vries-Burgers, Korteweg-de vries, Burgers, MOL, finite-difference

\section{INTRODUCTION}

A weakly nonlinear wave propagation in a prestressed fluid-filled stenosed elastic tube filled with a Newtonian fluid with variable viscosity fluid was studied by Gaik and Demiray (2008) by applying the reductive per-turbation method in long wave approximation. By using the stretched coordinate of boundary-value type and extending the field quantities into the asymptotic series of order $\varepsilon$ where $\varepsilon$ is a small parameter, the governing equations were reduced to the forced Korteweg-de Vries-Burgers (FkdVB) equation with varia-ble coefficients:

$$
\frac{\partial \mathrm{U}}{\partial \tau}+\mu_{1} \mathrm{U} \frac{\partial \mathrm{U}}{\partial \xi}-\mu_{2} \frac{\partial^{2} \mathrm{U}}{\partial \xi^{2}}+\mu_{3} \frac{\partial^{3} \mathrm{U}}{\partial \xi^{3}}+\mu_{4}(\tau) \frac{\partial \mathrm{U}}{\partial \xi}=\mu(\tau)
$$

Where:

$\mathrm{U}=$ The radial displacement

$\xi=$ A temporal variable

$\tau=$ A spatial variable

$\mu_{1}, \mu_{2}, \mu_{3}, \mu_{4}(\tau)$ and $\mu(\tau)$ are the coefficients of nonlinearity, dissipation, dispersion, variable coefficient and forcing term, respectively. The presence of forcing term and variable coefficient term, $\mu_{4}(\tau) U_{\xi}$ show the presence of ste-nosis. The dissipative term $-\mu_{2} U_{\xi \xi}$ in the FKdVB equation is caused by the effect of variable viscosity fluid. The coefficients of $\mu_{1}, \mu_{2}, \mu_{3}, \mu_{4}(\tau)$ and $\mu(\tau)$ were defined by Gaik and Demiray (2008) as:

$$
\begin{aligned}
& \mu_{1}=\frac{5}{2 \lambda_{\theta}}+\frac{\beta_{2}}{\beta_{1}}, \mu_{2}=\frac{\mathrm{v}}{2 \mathrm{c}} \mu_{3}=\frac{\mathrm{m}}{4 \lambda_{z}}+\frac{\lambda_{\theta}^{2}}{16}-\frac{\beta_{0}}{2 \beta_{1}} \\
& \mu_{4}(\tau)=\frac{\lambda_{\theta} \gamma_{2}}{\beta_{1}}(\mathrm{G})(\tau)-\left[\frac{\beta_{2}}{\beta_{1}}+\frac{1}{2 \lambda_{\theta}}\right] \mathrm{g}(\mathrm{r}) \\
& \mu(\tau)=\frac{1}{2} \mathrm{~g}^{\prime}(\tau)-\frac{\lambda_{\theta} \gamma_{1}}{2 \beta_{1}} \mathrm{G}^{\prime}(\tau)
\end{aligned}
$$

Where:

$$
\begin{aligned}
& \gamma_{0}=\frac{1}{\lambda_{\theta} \lambda_{z}}\left(\lambda_{\theta}-\frac{1}{\lambda_{\theta}^{3} \lambda_{z}^{2}}\right) \mathrm{F}\left(\lambda_{\theta}, \lambda_{z}\right) \\
& \gamma_{1}=\frac{1}{\lambda_{\theta} \lambda_{z}}\left[\left(1+\frac{3}{\lambda_{\theta}^{4} \lambda_{z}^{2}}\right)+2 \alpha\left(\lambda_{\theta}-\frac{1}{\lambda_{\theta}^{3} \lambda_{z}^{2}}\right)^{2}\right] \mathrm{F}\left(\lambda_{0}, \lambda_{z}\right) \\
& \gamma_{2}=\frac{1}{2 \lambda_{\theta} \lambda_{z}}\left[\begin{array}{l}
-\frac{12}{\lambda_{\theta}^{5} \lambda_{z}^{2}}+6 \alpha\left(\lambda_{\theta}-\frac{1}{\lambda_{\theta}^{3} \lambda_{z}^{2}}\right)\left(1+\frac{3}{\lambda_{\theta}^{4} \lambda_{z}^{2}}\right)+ \\
4 \alpha^{2}\left(\lambda_{\theta}-\frac{1}{\lambda_{\theta}^{3} \lambda_{z}^{2}}\right)^{3}
\end{array}\right] \mathrm{F}\left(\lambda_{\theta}, \lambda_{z}\right), \\
& \beta_{0}=\frac{1}{\lambda_{\theta}}\left(\lambda_{z}-\frac{1}{\lambda_{\theta}^{2} \lambda_{z}^{3}}\right) F\left(\lambda_{\theta}, \lambda_{z}\right), \beta_{1}=\gamma_{1}-\frac{\gamma_{\theta}}{\lambda_{\theta}} \\
& \beta_{2}=\gamma_{2}-\frac{\beta_{1}}{\lambda_{\theta}}
\end{aligned}
$$

given that:

$$
\mathrm{F}\left(\lambda_{\theta}, \lambda_{z}\right)=\exp \left[\alpha\left(\lambda_{\theta}^{2}+\lambda_{z}^{2}+\frac{1}{\lambda_{\theta}^{2} \lambda_{z}^{2}}-3\right)\right]
$$

Corresponding Author: K.G. Tay, Department of Communication Engineering, Faculty of Electrical and Electronic Engineering, 
$\alpha=1.948, \lambda_{\theta}=\lambda_{z}=1.6, v=1, \mathrm{c}=15.391, \mathrm{~m}=0.1, \mathrm{G}(\tau)=0$ and $g(\tau)=\operatorname{sech}(0.01 \tau)$. Here, $\alpha$ refers to material constant, $\lambda_{\theta}$ is the initial circumferential stretch ratio, $\lambda_{z}$ is the initial axial stretch ratio, $v$ is kinematic viscosity, $m$ is mass of artery, $\mathrm{c}$ is the scale parameter and $\mathrm{g}(\tau)$ is the stenosis function.

Note that when $\mu_{2}=0$, Eq. 1 becomes Forced Korteweg-de Vries (FKdV) equation arises in nonlinear wave propagation in a prestressed fluid-filled stenosed elastic tube filled with an invis-cid fluid derived by Gaik (2006):

$$
\frac{\partial \mathrm{U}}{\partial \tau}+\mu_{1} \mathrm{U} \frac{\partial \mathrm{U}}{\partial \xi}+\mu_{3} \frac{\partial^{3} \mathrm{U}}{\partial \xi^{3}}+\mu_{4}(\tau) \frac{\partial \mathrm{U}}{\partial \xi}=\mu(\tau)
$$

The FKdV Eq. 4 was solved numerically by using method of line and pseudospectral method (Tay et al., $2017 \mathrm{a}, \mathrm{b})$. When $\mu_{4}(\tau)=0$ and $\mu(\tau)=0$, Eq. 1 reduces to the standard Korteweg-de Vries-Burgers (KdVB) Eq. 5:

$$
\frac{\partial \mathrm{U}}{\partial \tau}+\mu_{1} \mathrm{U} \frac{\partial \mathrm{U}}{\partial \xi}-\mu_{2} \frac{\partial^{2} \mathrm{U}}{\partial \xi^{2}}+\mu_{3} \frac{\partial^{3} \mathrm{U}}{\partial \xi^{3}}=0
$$

The KdVB Eq. 5 describes shallow water waves on viscous fluid (Johnson, 1972) and wave propagation in elastic tube filled with a viscous fluid (Antar and Demiray, 1999). Notes that when $\mu_{2}=0$, Eq. 5 reduces to the standard Korteweg-de Vries (KdV) Eq. 6 :

$$
\frac{\partial \mathrm{U}}{\partial \tau}+\mu_{1} \mathrm{U} \frac{\partial \mathrm{U}}{\partial \xi}+\mu_{3} \frac{\partial^{3} \mathrm{U}}{\partial \xi^{3}}=0
$$

The KdV Eq. 6 was first introduced by Korteweg and Vries (De Vries and Korteweg, 1895) to describe the evolution of long, one-dimensional shallow water waves with small but finite amplitude. Zabusky and Kruskal (1965) discovered the concept of the solitons while studying the results of a numerical computation on the $\mathrm{KdV}$ equation. Since, then, the KdV equation has been found to describe many physical phenomena, including long internal waves in ocean, magneto hydrodynamics waves in warm plasma, ion acoustic waves in a plasma, acoustic waves on a crystal lattice (Ozis and Ozer, 2006) and wave propagation in an elastic tube filled with an inviscid fluid (Demiray, 2001):

$$
\frac{\partial \mathrm{U}}{\partial \tau}+\mu_{1} \mathrm{U} \frac{\partial \mathrm{U}}{\partial \xi}-\mu_{2} \frac{\partial^{2} \mathrm{U}}{\partial \xi^{2}}=0
$$

The Burgers Eq. 7 is a non-linear partial-differential equation which has a balance between the effects of nonlinearity and dissipation. It is a model for the traffic flow, turbulence, gas dy-namics, shock wave theory (Burger, 1948) and many other physical problems. Many studies have been devoted to the numerical solutions of the KdV Eq. 6, Burgers Eq. 7 and KdVB Eq. 5, however, none of the literature works dealt with numerical solutions of the FKdVB Eq. 1.

Motivated with the works of wave propagation which yielded the FKdVB Eq. 1 as well as numerical methods (Tay et al., 2017a, b; Tay et al., 2018), we are going to find numerical solution of the FkdVB by two numerical methods, namely Method of Lines (MOL) and finitedifference method. The numerical solution of the FKdVB Eq. 1 is then compared in terms of its maximum absolute error at certain space $\tau$ with progressive wave solution conducted by Gaik and Dimroy (2008).

\section{MATERIALS AND METHODS}

The MOL: The MOL is a powerful method used to solve Partial Differential Equations (PDEs). By replacing the spatial derivatives using finite-difference approximation, the PDEs will be reduced into time dependent system of ODEs. These system of ODEs can be solved by using techniques of solving initial value problem of ODEs such as the fourth-order Runge-Kutta (RK4) method.

MOL has been widely used to solve the nonlinear evolution equa-tions such as Korteweg-de Vries (KdV) equation (Schiesser, 1994), extended nonlinear KdV equation, good Boussinesq equation, fifth-order Kaup-Kupershmidt equation and an extended fifth-order Korteweg-de Vries (KdV5) equation (Saucez et al., 2004), delay differential equations (Koto, 2004), two-dimensional sine-Gordon equation (Bratsos, 2007), the Nwogu one-dimensional extended Boussinesq equation (Hamdi et al., 2005).

In this study, the temporal derivatives $\xi$ in Eq. 1 were firstly discre-tized using central finite difference formulae as follows:

$$
\begin{aligned}
& \mathrm{U}_{\xi} \approx \frac{\mathrm{U}_{\mathrm{i}+1}-\mathrm{U}_{\mathrm{i}-1}}{2 \Delta} \\
& \mathrm{U}_{\xi \xi} \approx \frac{\mathrm{U}_{\mathrm{i}+1}-2 \mathrm{U}_{\mathrm{i}}+\mathrm{U}_{\mathrm{i}-1}}{(\Delta \xi)^{3}} \\
& \mathrm{U}_{\xi \xi \xi} \approx \frac{\mathrm{U}_{\mathrm{i}+2}-2 \mathrm{U}_{\mathrm{i}+1}+2 \mathrm{U}_{\mathrm{i}-1}-\mathrm{U}_{\mathrm{i}-2}}{2(\Delta \xi)^{3}}
\end{aligned}
$$


$i$ is the index denoting the temporal position along $\xi$-axis and $\Delta \xi$ is the step size along the $\xi$-axis. The $\xi$-interval is divided into $\mathrm{M}$ points with $\mathrm{i}=1,2, \ldots, \mathrm{M}-1, \mathrm{M}$. Therefore, the MOL ap-proximation of Eq. 1 is given by:

$$
\begin{aligned}
& \frac{\partial \mathrm{U}_{\mathrm{i}}}{\partial \tau}=\frac{\mu_{1}}{2 \Delta \xi} \mathrm{U}_{\mathrm{i}}\left(\mathrm{U}_{\mathrm{i}+1}-\mathrm{U}_{\mathrm{i}-1}\right)+\frac{\mu_{2}}{(\Delta \xi)^{2}}\left(\mathrm{U}_{\mathrm{i}+1}-2 \mathrm{U}_{\mathrm{i}}+\mathrm{U}_{\mathrm{i}-1}\right)- \\
& \frac{\mu_{3}}{2(\Delta \xi)^{3}}\left(\mathrm{U}_{\mathrm{i}+2}-2 \mathrm{U}_{\mathrm{i}+1}+2 \mathrm{U}_{\mathrm{i}-1}-\mathrm{U}_{\mathrm{i}-2}\right) \\
& \frac{\mu_{4}(\tau)}{2 \Delta \xi}\left(\mathrm{U}_{\mathrm{i}+1}-\mathrm{U}_{\mathrm{i}-1}\right)+\mu(\tau) \equiv \mathrm{f}\left(\mathrm{U}_{\mathrm{i}}\right)
\end{aligned}
$$

Since, there is only one independent variable which is $\tau$, hence, Eq. 9 is an ODE. Besides, since, $i$ varies from 1, 2, up to $M$, thus, Eq. 9 represents a system of $M$ equations of $\mathrm{ODEs}$ with the initial condition given by:

$$
\mathrm{U}\left(\xi_{\mathrm{i}}, \tau=0\right) \mathrm{U}_{0}\left(\xi_{\mathrm{i}}\right), \mathrm{i}=1,2, \ldots, \mathrm{M}-1, \mathrm{M}
$$

For the space integration, the RK4 method is applied. Thus, the numerical solution at space $\tau_{j+1}$ is:

$$
\mathrm{U}_{\mathrm{i}}^{j+1}=\mathrm{U}_{\mathrm{i}}^{\mathrm{j}}+\frac{1}{6}\left(\mathrm{a}_{\mathrm{i}}^{\mathrm{j}}+2 \mathrm{~b}_{\mathrm{i}}^{\mathrm{j}}+2 \mathrm{c}_{\mathrm{i}}^{\mathrm{j}}+\mathrm{d}_{\mathrm{i}}^{\mathrm{j}}\right)
$$

Where:

$$
\left.\begin{array}{l}
\mathrm{a}_{\mathrm{i}}^{\mathrm{j}}=\Delta \tau \mathrm{f}\left(\mathrm{U}_{\mathrm{i}}^{\mathrm{j}}\right), \\
\mathrm{b}_{\mathrm{i}}^{\mathrm{j}}=\Delta \tau \mathrm{f}\left(\mathrm{U}_{\mathrm{i}}^{\mathrm{j}}+\frac{1}{2} \mathrm{a}_{\mathrm{i}}^{\mathrm{j}}\right), \\
\mathrm{c}_{\mathrm{i}}^{\mathrm{j}}=\Delta \tau \mathrm{f}\left(\mathrm{U}_{\mathrm{i}}^{\mathrm{j}}+\frac{1}{2} \mathrm{~b}_{\mathrm{i}}^{\mathrm{j}}\right), \\
\mathrm{d}_{\mathrm{i}}^{\mathrm{j}}=\Delta \tau \mathrm{f}\left(\mathrm{U}_{\mathrm{i}}^{1}+\mathrm{c}_{\mathrm{i}}^{\mathrm{j}}\right)
\end{array}\right\}
$$

Here, $\Delta \tau$ is the step size of the spatial coordinate.

The finite-difference method: The temporal and spatial coordinates $(\xi, \tau)$ in Eq. 1 are discretized as coordinate grid as $\left(\xi_{\mathrm{i}}, \tau_{\mathrm{j}}\right)$ where $\xi_{\mathrm{i}}=-\mathrm{L}+2(\mathrm{i}-1) \mathrm{L} / \mathrm{N}, \tau_{\mathrm{j}}=(\mathrm{j}-1) \Delta \tau$. Here, $\Delta \tau$ is the step size in spatial coordinate, $\mathrm{i}=1,2,3, \ldots, \mathrm{N}, \mathrm{N}+1$, $\mathrm{j}=1,2,3, \mathrm{M}, \mathrm{M}+1, \mathrm{~N}$ and $\mathrm{M}$ are the number of intervals in the temporal and spatial coordinates, respectively. $\mathrm{L}$ is half interval of temporal coordinates, respectively.

The FKdVB Eq. 1 is approximated at the point $\left(\xi_{i}, \tau_{j+1 / 2}\right)$. The 3-point central difference approximation on space $\tau$ and time $\xi$ were used. The spatial derivatives of Eq. 1 at $\left(\xi_{\mathrm{i}}, \tau_{\mathrm{j}+1 / 2}\right)$ is approximated by averaging the approximation at the point $\left(\xi_{\mathrm{i}}, \tau_{\mathrm{j}+1}\right)$ and $\left(\xi_{i}, \tau_{j}\right)$ follows (Helal and Mehanna, 2006) Eq. 1 becomes:

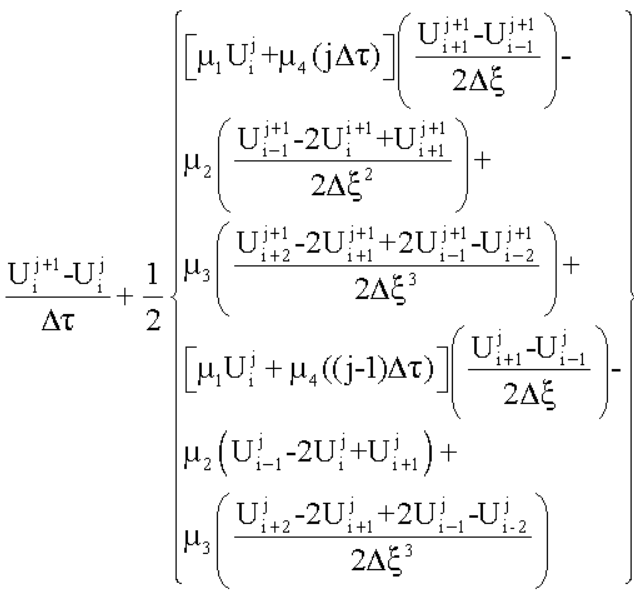

$$
\begin{aligned}
& =\frac{1}{2}[\mu(\mathrm{j} \Delta \tau)+\mu((\mathrm{j}-1) \Delta \tau)]
\end{aligned}
$$

Here, $\Delta \xi$ the step sizes in the temporal coordinates. Simplifying and collecting the terms lead to the following implicit scheme:

$$
\begin{aligned}
& -r \mu_{3} U_{i-2}^{j+1}+a_{1} U_{i-1}^{j+1}+a_{2} U_{i}^{j+1}+a_{3} U_{i+1}^{j+1}+r \mu_{3} U_{i+2}^{j+1}= \\
& r \mu_{3} U_{i-2}^{j}+b_{1} U_{i-1}^{j}+b_{2} U_{i}^{j}+b_{3} U_{i+1}^{j}-r \mu_{3} U_{i+2}^{j}+g
\end{aligned}
$$

Where:

$$
\begin{aligned}
& \mathrm{a}_{1}=-\mathrm{r} \Delta \xi^{2}\left[\mu_{1} \mathrm{U}_{\mathrm{i}}^{\mathrm{j}}+\mu_{4}(\mathrm{j} \Delta \tau)\right]-2 \mathrm{r} \Delta \xi \mu_{2}+2 \mathrm{r} \mu_{3} \\
& \mathrm{a}_{2}=4+4 \mathrm{r} \Delta \xi \mu_{2} \\
& \mathrm{a}_{3}=\mathrm{r} \Delta \xi^{2}\left[\mu_{1} \mathrm{U}_{\mathrm{i}}^{\mathrm{j}}+\mu_{4}(\mathrm{j} \Delta \tau)\right]-2 \mathrm{r} \Delta \xi \mu_{2}+2 \mathrm{r} \mu_{3} \\
& \mathrm{~b}_{1}=\mathrm{r} \Delta \xi^{2}\left[\mu_{1} \mathrm{U}_{\mathrm{i}}^{\mathrm{j}}+\mu_{4}((\mathrm{j}-1) \Delta \tau)\right]+2 \mathrm{r} \Delta \xi \mu_{2}-2 \mathrm{r} \mu_{3} \\
& \mathrm{~b}_{2}=4-4 \mathrm{r} \Delta \xi \mu_{2} \\
& \mathrm{~b}_{3}=-\mathrm{r} \Delta \xi^{2}\left[\mu_{1} \mathrm{U}_{\mathrm{i}}^{\mathrm{j}}+\mu_{4}((\mathrm{j}-1) \Delta \tau)\right]+2 \mathrm{r} \Delta \xi \mu_{2}+2 \mathrm{r} \mu_{3} \\
& \mathrm{~g}=2 \mathrm{r} \Delta \xi^{3}[\mu(\mathrm{j} \Delta \tau)+\mu((\mathrm{j}-1) \Delta \tau)] \\
& \mathrm{r}=\frac{\Delta \tau}{\Delta \xi^{3}}
\end{aligned}
$$

Progressive wave (Exact) solution: The progressive wave solution of the FKdVB equation as given by Gaik and Demiray (2008):

$$
\begin{aligned}
& \mathrm{u}=\frac{\mathrm{a}}{\mu_{1}}+\frac{3 \mu_{2}^{2}}{25 \mu_{1} \mu_{3}}\left(\operatorname{sech}^{2} \zeta-2 \tanh \zeta\right) \\
& +\frac{1}{2}\left[\mathrm{~g}(\tau)-\frac{\lambda_{\theta} \lambda_{1}}{\beta_{1}} \mathrm{G}(\tau)\right]
\end{aligned}
$$

where, a is a constant. The phase function $\zeta$ can be expressed as: 


$$
\zeta=\frac{\mu_{2}}{10 \mu_{3}}\left\{\xi-\mathrm{a \tau}-\int_{0}^{\tau}\left[\left(\frac{3}{4 \lambda_{\theta}}-\frac{\beta_{2}}{2 \beta_{1}}\right) \mathrm{g}(\mathrm{s})+\frac{\lambda_{\theta}}{\beta_{1}}\left(\gamma_{2}-\frac{\mu_{1} \gamma_{1}}{2}\right) \mathrm{G}(\mathrm{s})\right] \mathrm{ds}\right\}
$$

\section{RESULTS AND DISCUSSION}

For both numerical methods, we need the initial condition to start the numerical simulations. By letting $\tau$ $=0$ in Eq. 16 and $\mathrm{a}=2$, we used the initial condition as:

$$
\begin{aligned}
& \mathrm{U}(\xi, 0)=\frac{\mathrm{a}}{\mu_{1}}+\frac{3 \mu_{2}^{2}}{25 \mu_{1}, \mu_{3}} \operatorname{sech}^{2}\left(\frac{\mu_{2}}{10 \mu_{3}} \xi\right) \\
& -\frac{6 \mu_{2}^{2}}{25 \mu_{1}, \mu_{3}} \tanh \left(\frac{\mu_{2}}{10 \mu_{3}} \xi\right)+0.5
\end{aligned}
$$

To calculate the accuracy of the numerical solution with the pro-gressive wave solution, the maximum absolute errors between the progressive wave and numerical solutions were calculated based on the Eq. 19:

$$
\mathrm{L} \infty={ }_{\max }\left|\mathrm{U}_{\text {progressive }}-\mathrm{U}_{\text {numerical }}\right|
$$

By utilizing $\Delta \xi=0.1, \Delta \tau=0.001$ in the MOL scheme, we obtained the MOL solution of the FKdVB Eq. 1 with time $\xi$ at certain space $\tau$ as displayed in Fig. 1a. On the other hand, Fig. $1 \mathrm{~b}$ represents the progressive wave

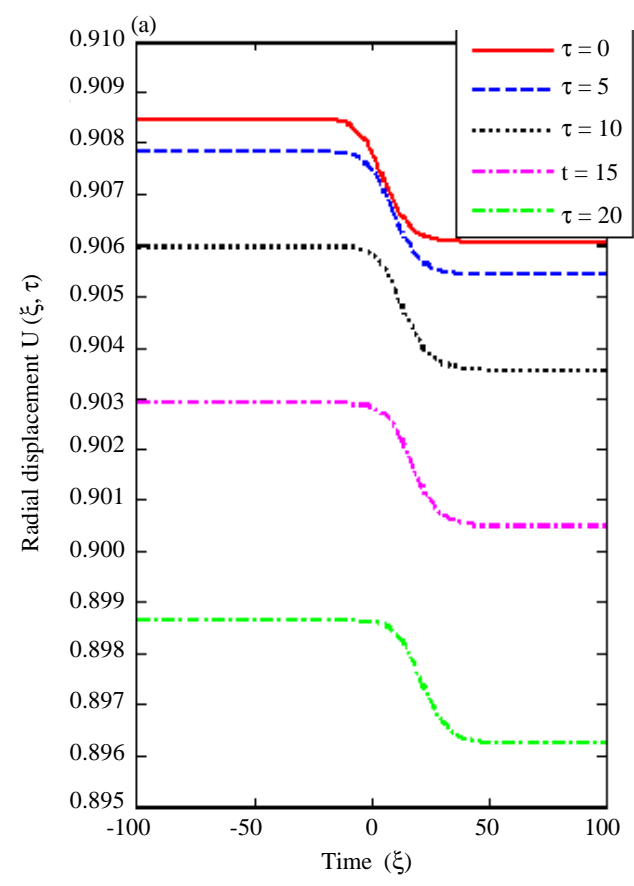

solution of the FKdVB Eq. 1 with time parameter $\xi$ at certain space $\tau$. The solution of the FKdVB Eq. 1 with time $\xi$ shows a decreasing shock profile propagating to the right with a decrease in wave amplitude as space $\tau$ increases. Intuitively, both Fig. 1a and $\mathrm{b}$ are identical.

To check if both Fig. 1a and $\mathrm{b}$ are indistinguishable, both MOL and progressive wave solutions were plotted on the same figure. It can be seen that both MOL and progressive wave solutions are exactly the same in terms of amplitude and its position as shown in Fig. 2 as they are overlapped exactly intuitively.

To confirm, if there is any numerical error, the absolute errors between MOL and progressive wave solutions were plotted in Fig. 3. It is worth noting that there are still some small errors even both MOL and progressive wave solutions were overlapped exactly intuitively.

We then compute the maximum absolute error between the progressive wave and MOL solutions for each discretized time $\xi$ point at certain space $\tau$ based on the Eq. 19 as given in Table 1. It shows that most of the maximum absolute errors are in order of $10^{-7}$.

Table 1: Maximum absolute error of the FKdVB equation for different

\begin{tabular}{ll}
\multicolumn{2}{c}{ space $\tau$ by MOL method } \\
\hline Space $(\tau)$ & $\mathrm{L}_{\mathrm{s}_{0}}$ \\
\hline 0 & 0 \\
5 & $1.3592 \times 10^{7}$ \\
10 & $2.7 \times 10^{-7}$ \\
15 & $4.0103 \times 10^{7}$ \\
20 & $5.2739 \times 10^{7}$ \\
\hline
\end{tabular}

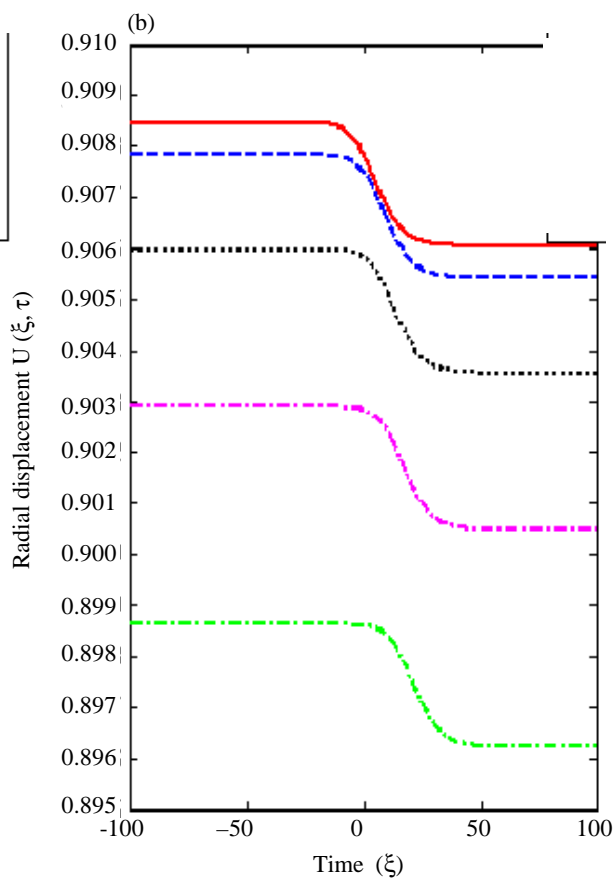

Fig. 1: a) MOL and b) Progressive wave solutions of the FKdVB equation versus time $\xi$ for different space $\tau$ with $\Delta \xi$ $=0.1, \Delta \tau=0.001$ 
Next, by using $0.1,0.01 \Delta \xi=0.1, \Delta \tau=0.01$ in the finite-difference scheme, we obtained the finitedifference solution of the FKdVB Eq. 1 with time $\xi$ at certain space $\tau$ as given in Fig. 4a while Fig. $4 \mathrm{~b}$ shows the progressive wave solution of the FKdVB Eq. 1 with temporal parameter $\xi$ at certain space $\tau$. Both Fig. $4 \mathrm{a}$ and $\mathrm{b}$ are synonymous.

To confirm, if both the finite-difference and progressive wave solutions are similar, both the finite-difference and progressive wave solutions were plotted on the same graph as pictured in Fig. 5. Noteworthy, Fig. 5 shows there is no difference between both solutions as both graphs are overlapped exactly.

Similarly to check, if there is any error between finite-difference and progressive wave solutions, the absolute errors between the finite-difference scheme and progressive wave solution were de-picted in Fig. 6. Surprisingly, there are still some small errors between finite-difference and progressive wave solutions.

On the other hand, the maximum absolute error between the pro-gressive wave and finite-difference solutions for each temporal point at certain space were calculated based on the Eq. 19 and tabulated in Table 2. It shows that the maximum absolute errors are in order of $10^{-8}$.

Table 2: Maximum absolute error of the FKdVB equation for different space

\begin{tabular}{ll}
\multicolumn{2}{c}{$\tau$ by finite-difference method } \\
\hline Space $(\tau)$ & $\mathrm{L}_{c o}$ \\
\hline 0 & 0 \\
5 & $1.0774 \times 10^{8}$ \\
10 & $1.8209 \times 10^{8}$ \\
15 & $2.2417 \times 10^{8}$ \\
20 & $2.8955 \times 10^{8}$ \\
\hline
\end{tabular}

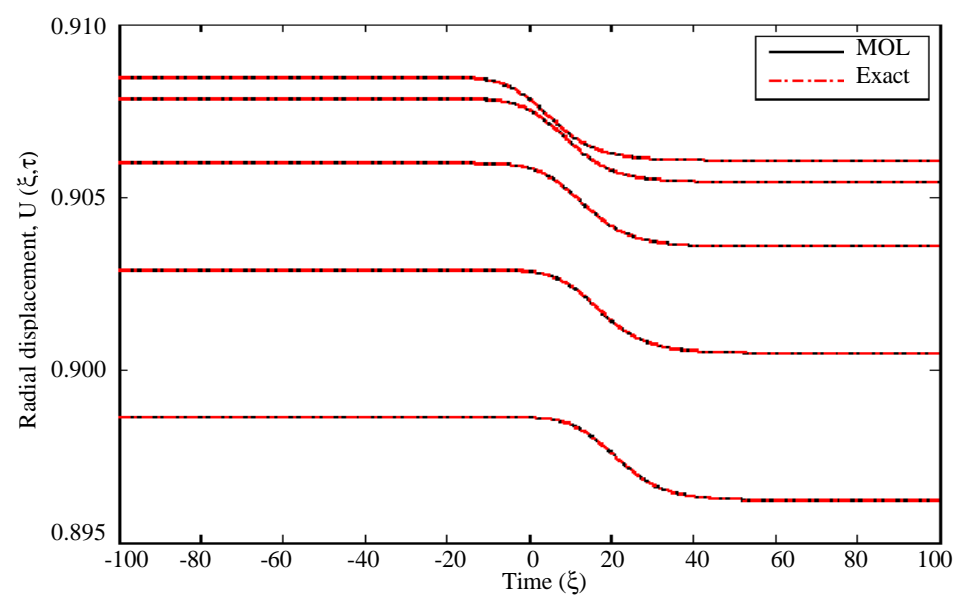

Fig. 2: MOL and progressive wave solutions of the FKdVB equation versus time $\xi$ for different space $\tau$

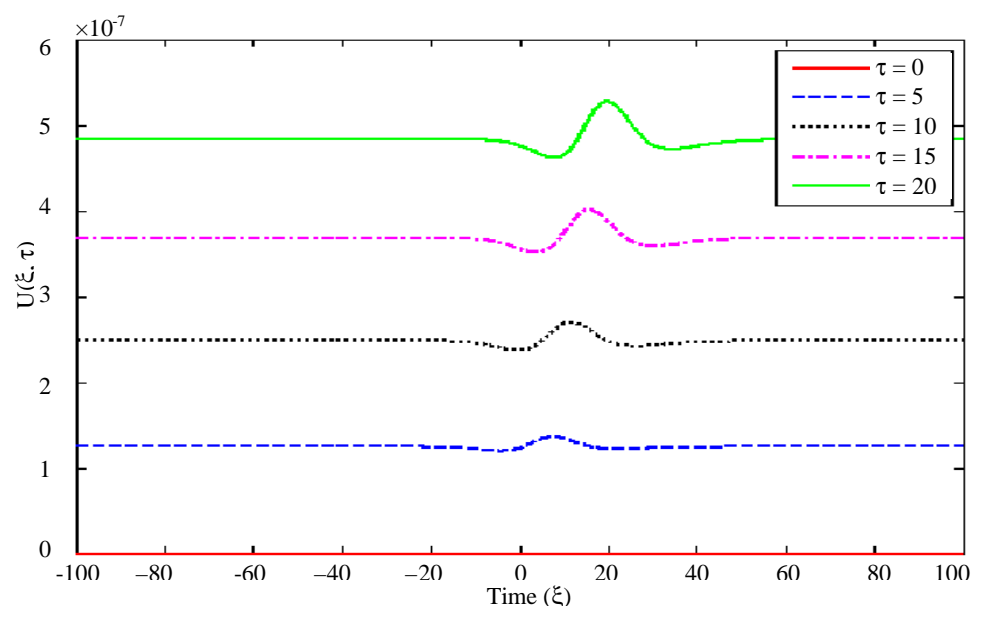

Fig. 3: Absolute error between MOL and progressive wave solutions of the FKdVB equation versus time $\xi$ for different space $\tau$ 
(a)

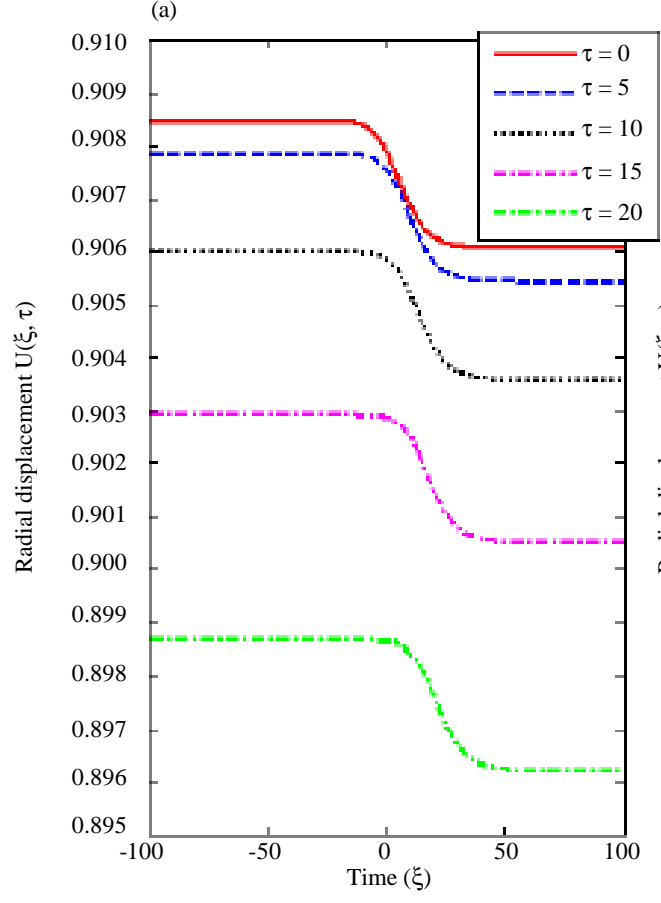

(b)

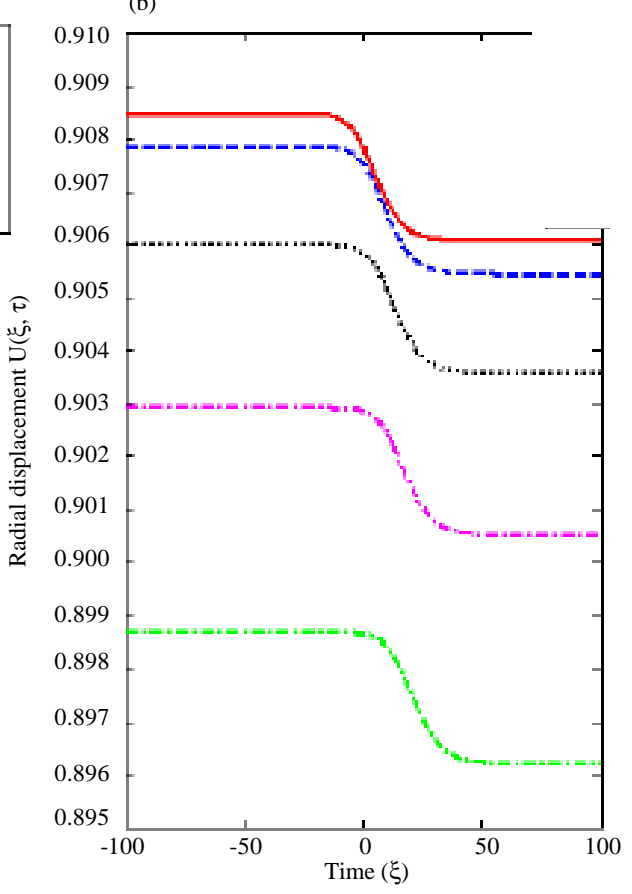

Fig. 4: a) Finite-difference and b) Progressive wave solutions of the FKdVB equation versus time $\xi$ for different space with $\Delta \xi=0.1, \Delta \tau=0.01$

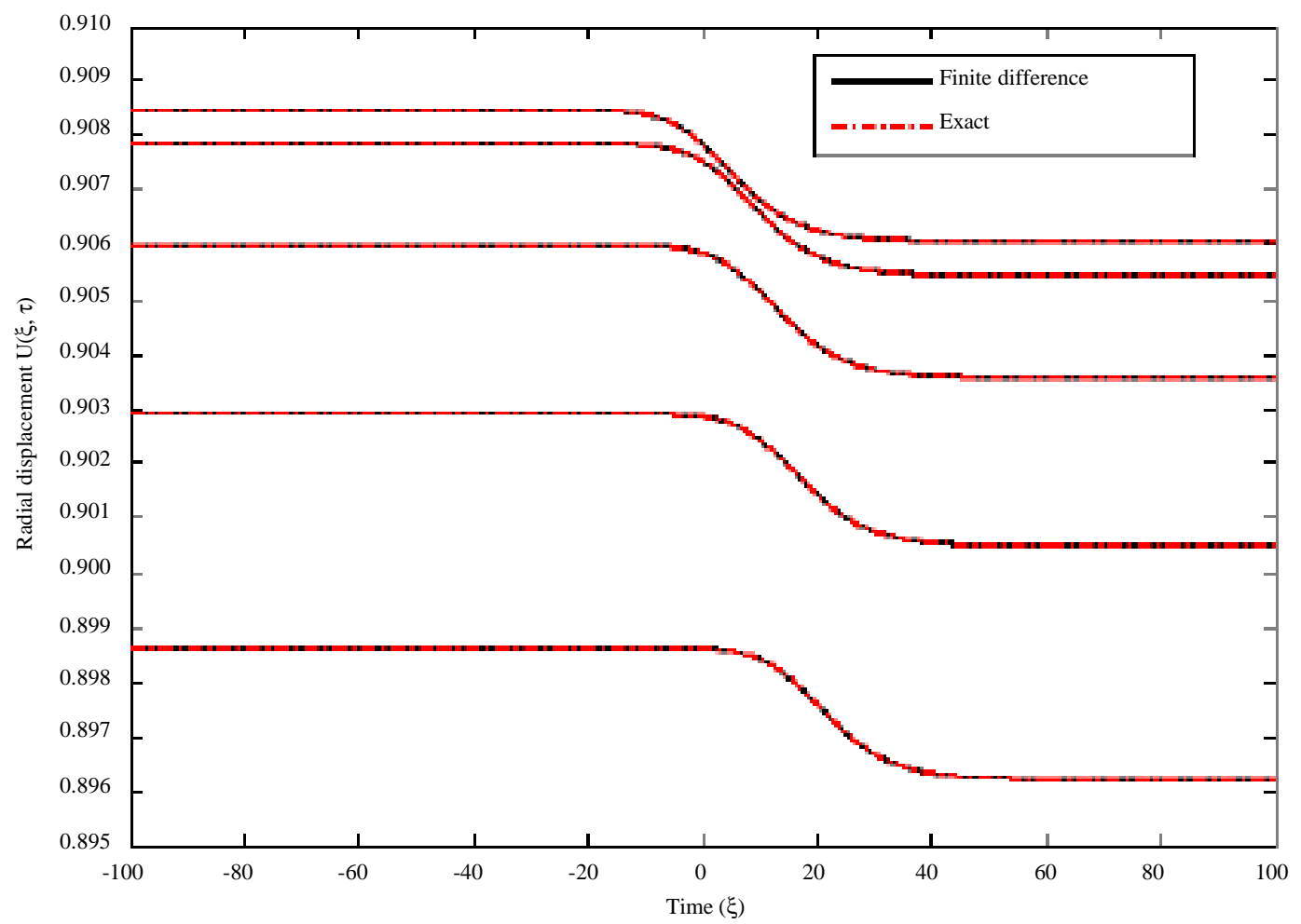

Fig. 5: Finite-difference and progressive wave solutions of the FKdVB equation versus time $\xi$ for different space $\tau$ 


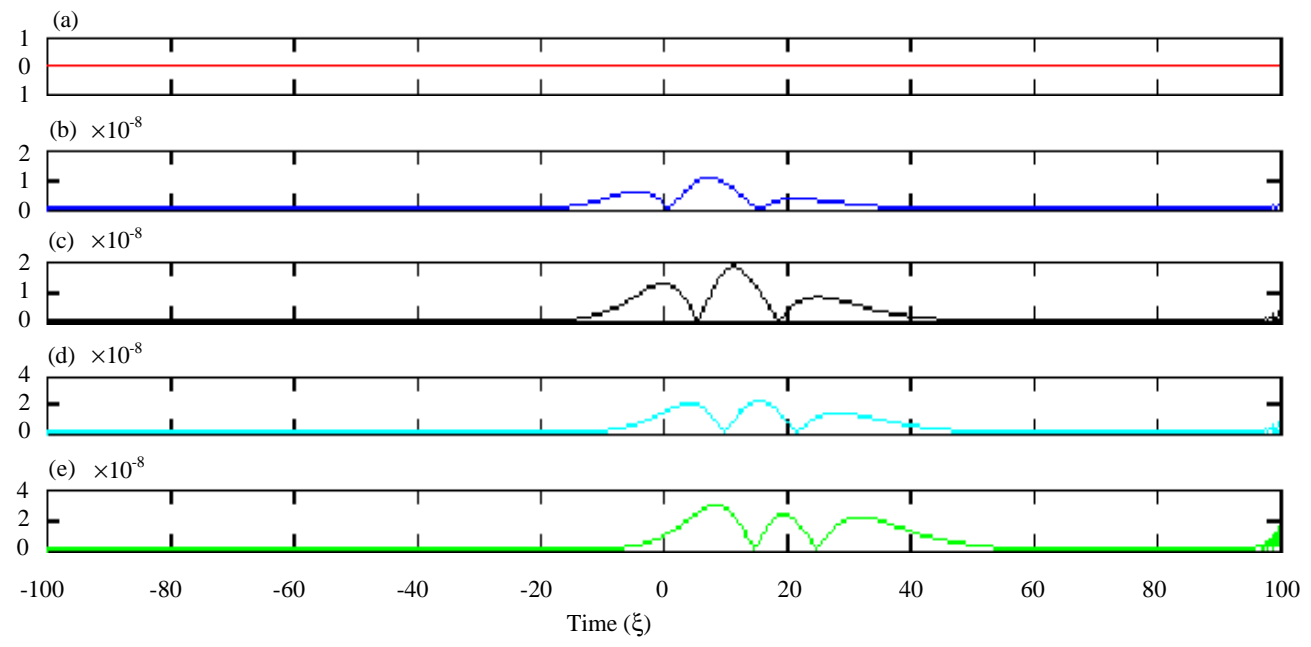

Fig. 6: Absolute error between finite-difference and progressive wave solutions of the FKdVB equation versus time $\xi$ for different space $\tau$ : a) Space $\tau=0$; b) Space $\tau=5$; c) Space $\tau=10$; d) Space $\tau=15$ and e) Space $\tau=20$

\section{CONCLUSION}

We have solved the FKdVB Eq. 1 by using the MOL and finite-difference method. The MOL and finite-difference solutions of the FKdVB Eq. 1 were plotted versus the progressive wave solution. From the observation, it was found that there were no differences for both MOL, finite-difference and progressive wave solutions. MOL and finite-difference method can solve the FkdVB Eq. 1 pretty well with maximum absolute error in order of $10^{-7}$ and $10^{-8}$, respectively. Hence, finite-difference solves the FKdVB better, since, it gives smaller maximum absolute error, if compared to MOL method.

\section{ACKNOWLEDGEMENT}

We would like to thank UTHM Research fund, UTHM Tier 12018 research grant vote number $\mathrm{H} 258$ and Fundamental Research Grant Scheme (FRGS) vot K095 granted by Ministry of Education (MOE) Malaysia for financial support of this project and fundamental research Grant scheme (FRGS) vot Ko93 granted by ministry of education (Moe) Malaysia.

\section{REFERENCES}

Antar, N. and H. Demiray, 1999. Weakly nonlinear waves in a prestressed thin elastic tube containing a viscous fluid. Intl. J. Eng. Sci., 37: 1859-1876.

Bratsos, A.G., 2007. The solution of the two-dimensional sine-Gordon equation using the method of lines. J. Comput. Appl. Math., 206: 251-277.

Burgers, J.M., 1948. A mathematical model illustrating the theory of turbulence. Adv. Applied Mech., 11: 171-199.
De Vries, G. and D.J. Korteweg, 1895. On the change of form of long waves advancing in a rectangular canal and on a new type of long stationary waves. Phil. Mag., 39: 422-443

Demiray, H., 2001. Solitary waves in fluid-filled elastic tubes: Weakly dispersive case. Intl. J. Eng. Sci., 39: 439-451.

Gaik, T.K. and H. Demiray, 2008. Forced korteweg-de vries-burgers equation in an elastic tube filled with a variable viscosity fluid. Chaos Solitons Fractals, 38: 1134-1145.

Gaik, T.K., 2006. Forced Korteweg-de Vries equation in an elastic tube filled with an inviscid fluid. Intl. J. Eng. Sci., 44: 621-632.

Gaik, T.K., Y.Y. Choy, W.K. Tionng and C.T. Ong, 2018. Numerical solutions of the dissipative nonlinear Schrodinger equation with variable coefficient arises in elastic tube. Dyn. Continuous Discrete Impulsive Syst. Ser. B. Appl. Algorithms, 25: 53-61.

Hamdi, S., W.H. Enright, Y. Ouellet and W.E. Schiesser, 2005. Method of lines solutions of the extended Boussinesq equations. J. Comput. Appl. Math., 183: 327-342.

Helal, M.A. and M.S. Mehanna, 2006. A comparison between two different methods for solving KdV-burgers equation. Chaos, Solitons Fractails, 28: 320-326.

Johnson, R.S., 1972. Shallow water waves on a viscous fluid-the undular bore. Phys. Fluids, 15: 1693-1699.

Koto, T., 2004. Method of lines approximations of delay differential equations. Comput. Math. Appl., 48: 45-59. 
Ozis, T. and S. Ozer, 2006. A simple similarity-transformation-iterative scheme applied to Korteweg-de Vries equation. Applied Math. Comput., 173: 19-32.

Saucez, P., A.V. Wouwer, W.E. Schiesser and P. Zegeling, 2004. Method of lines study of nonlinear dispersive waves. J. Comput. Appl. Math., 168: 413-423.

Schiesser, W.E., 1994. Method of lines solution of the Korteweg-de Vries equation. Comput. Math. Appl., 28: $147-154$.
Tay, K.G., W.K. Tiong, Y.Y. Choy and C.T. Ong, $2017 \mathrm{~b}$. Method of lines and pseudospectral solutions of the forced Korteweg-de Vries equation with variable coefficients arises in elastic tube. Intl. J. Pure Appl. Math., 116: 985-999.

Tay, K.G., Y.Y. Choy, W.K. Tiong, C.T. Ong and N.M. Yazid, 2017a. Numerical solutions of the forced perturbed korteweg-de vries equation with variable coefficients. Intl. J. Pure Appl. Math., 112: 557-569.

Zabusky, N.J. and M.D. Kruskal, 1965. Interaction of solitons in a collisionless plasma and the recurrence of initial states. Phys. Rev. Let., 15: 240-243. 